\title{
Comparative Analysis of Different Data Dissemination Techniques based Genetic Algorithm and Fuzzy in Vehicular Adhoc Networks (VANETs)
}

\author{
Bhawna Dhawan \\ M.Tech Research Scholar \\ Deptt. Of CSE \\ PTU Regional Centre \\ ACET, Amritsar
}

\author{
TanuPreet Singh,PhD \\ HOD ECE \\ Deptt. of ECE \\ PTU Regional Centre \\ ACET, Amritsar
}

\begin{abstract}
In today's world Vehicular adhoc network done promising job towards public safety and provide important element to the transport facility. A Vehicular adhoc network is a new technology which has garnered enormous attention in recent years.VANETs is a special class of MANETs which uses vehicles as a mobile node. It uses the Intelligent transportation system in which vehicles can communicate with each other to avoid large number of problems such as real-time traffic problem, parking availability problem etc. The communication among the vehicles is at greater risk because the messages are broadcasted by wireless channel and vehicles move with high mobility. VANET does not have any fixed infrastructure Data dissemination is the tough job among these vehicles. While driving, a large amount of data and information are accessible to everyone. Many attractive applications over vehicular ad hoc network (VANETs) need data to be transmitted to the remote destinations through multiple paths, but some unique characteristics of VANETs incur unstable data delivery performances. Data dissemination in VANETs is more challenging because vehicles are highly mobile. Efficient data dissemination to a desired number of receivers in a vehicular ad hoc network (VANET) is a new issue and a challenging one considering the dynamic nature of VANETs. To overcome such situation and achieve efficient data dissemination among these vehicles different techniques are used. This paper represents a simple and robust dissemination technique that efficiently deals with data dissemination where the density of roadside base stations and vehicles distribution are both high. This technique divides the users in two categories premium user as well as free users. This paper illustrates three schemes such as fuzzy inference system, genetic algorithm scheme and hybrid of fuzzy inference and genetic algorithm. Two types of users have been taken in this paper.
\end{abstract}

\section{General Terms}

VANETs, Data Dissemination

\section{Keywords}

Data Dissemination, Fuzzy, Genetic Algorithm, HFGA

\section{INTRODUCTION}

The recent advances in technologies and in the standardization of the elements that compound the Intelligent Transportation System bring new attention on the theme of vehicles to vehicles communication for smarter and safer road traffic [1]. VANET is a new technology that combines the potentials of new generation wireless networks into vehicles. During the last couple of years, advancement in embedded systems and wireless technologies has extended into new domains. Intelligent transportation systems (ITS) are taking information and communications technology capabilities and advantages to stimulate vehicle networks into new types of vehicular ad hoc networks (VANETs) [2]. The main goal of a vehicular ad hoc network system is to enable efficient data dissemination for drivers and passengers safety and comfort and to protect them from danger [3]. VANET focuses to offer (i) continuous connectivity for mobile users while they are on the road, which enables them to link with other users through the latter's home or office based networks and (ii) efficient wireless connection between vehicles without access to any fixed infrastructure which enables ITS. VANET is also known as inter-vehicle communication (IVC). VANET devices such as on -board units are fixed in vehicles and functions as the nodes to transmit and receive messages through wireless networks. These devices provide drivers and passengers with the latest information on accidents, flooding, rain, traffic etc. By obtaining such information on time, drivers can make appropriate decisions and avoid misfortunes. Altering drivers about the conditions of roads, traffic and related aspects are crucial to safety and to the regulation of vehicle flow. In future vehicular networks, the requirements for communication between vehicles and the outside world will only increase. The communication protocol that is used as a standard to support wireless access in vehicular environment (WAVE) was recently defined by the ITS IEEE 802.11p Task Group [4]. Communication modules are expected to be embedded in vehicular entertainment systems, integrating the Microsoft Silver-light streaming technique and Microsoft Expression Encoder to deliver Smooth Streaming effect and provide users with a high-quality viewing experience [5]. VANET enables participating vehicles to communicate cooperatively for exchanging information about road conditions and travel situations, by providing a selforganizing network environment, without requiring a fixed infrastructure or centralized administration [6]. With the increasing number of vehicles being equipped with communication capabilities, large scale VANETs are expected to be available in the near future [7]. Existing VANET dissemination techniques can be classified into three models: push, pull and hybrid. In the push model, data is disseminated proactively using periodic broadcast, while in the pull model, data is disseminated on-demand [8], where a routing protocol carries data to relatively faraway distances. There are few schemes that combine both dissemination 
models together so as to support different types of applications.

\subsection{Data dissemination techniques}

Different data dissemination techniques for VANETs are proposed to fit in different applications. Data Dissemination is the communication between the vehicles and roadside base station. Vehicles can access the data through $\mathrm{V} 2 \mathrm{~V}$ technique or V2I technique. These vehicles can obtain information from the roadside base station as well as nearby vehicles. Each vehicle communicates with nearby vehicles in a highly dynamic ad hoc networking environment through V2V communication. In this paper, two techniques have been taken under consideration, which are as follows:

V2I/I2V Dissemination: It consists of two type's data dissemination- push based and pull based. In push based data dissemination, the data can be efficiently delivered from the moving vehicles or fixed base station (RSU) to another vehicle, it is mostly used in the traffic conditions, e-advertisement. While in pull based data dissemination any vehicle is enabled to query information about specific location or target it is form of request and respond type model. Mainly used in enquiry about the parking lot, nearby coffee shop basically non-popular data which user specific [9] [10].

ii) V2V Dissemination: In vehicle to vehicle data dissemination, data transmit to the vehicle by another vehicle. If vehicle is out of range of cluster of Base station then base station sends the data to the vehicle through another vehicle.

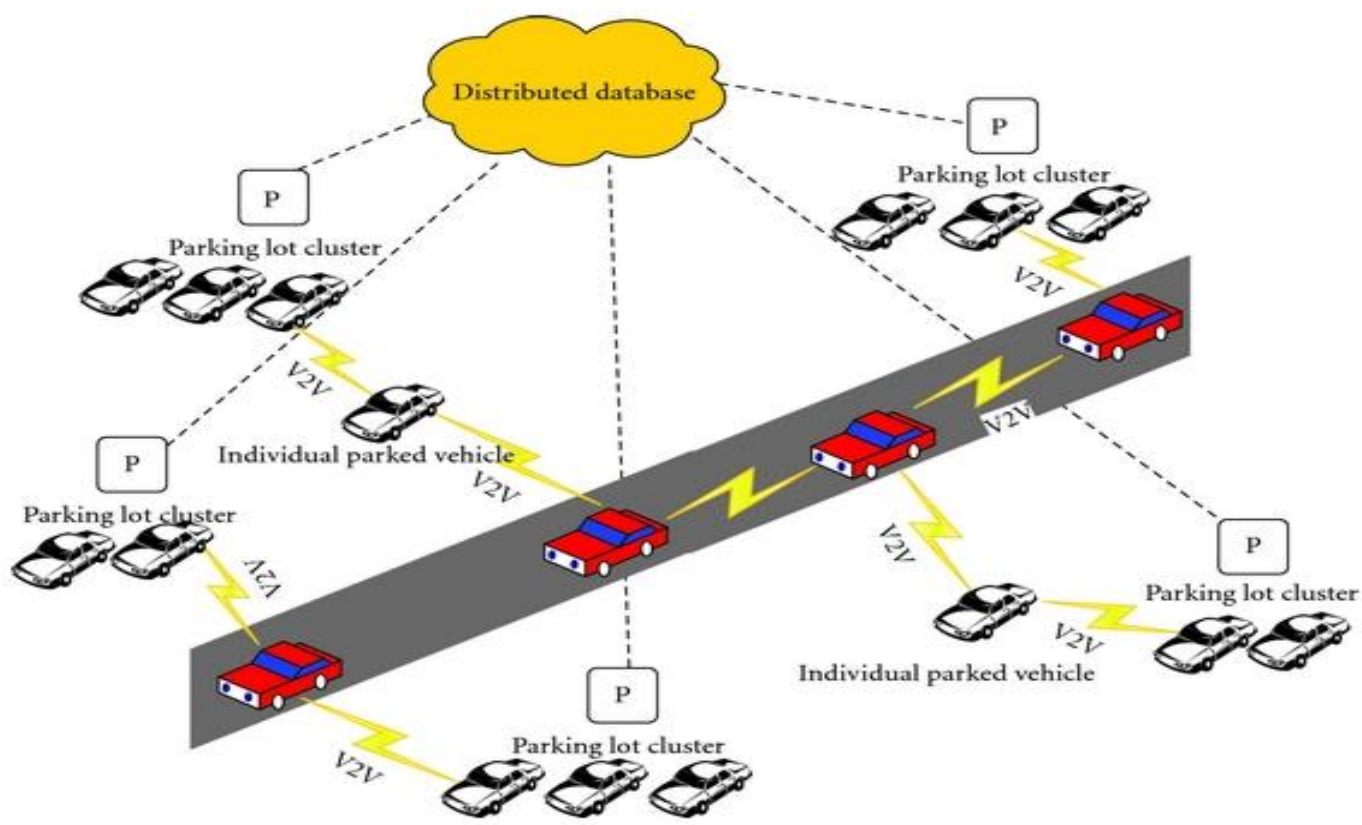

Fig1: VANET [11]

\subsection{Fuzzy Inference System}

Fuzzy set theory founded by Zadeh[12] is a paradigm which gained interest of many researchers, specially in the area of control theory. Its main idea consists in modeling the reasoning process of an expert who uses linguistic terms rather than numbers for controlling a complex plant. Granularity of knowledge is a characteristic feature of fuzzy inference systems which is attained by means of membership functions defined on a domain of interest. A typical fuzzy decision rule, containing linguistic terms connected by fuzzy operators, is a generalization of a classic crisp decision rule [13]. An application of fuzzy inference systems is to find out the optimal path and best vehicle to send the data to other vehicles. It not only providing an adequate system of decision rules, but also involves a correct choice of membership functions of linguistic terms and selecting a defuzzification method. In the present paper, we use a Tsukamoto defuzzification method. An important issue in application of fuzzy inference systems is interpretation, readability and comprehensibility of the decision process [especially in the case of large systems of decision rules with many linguistic terms [14-16]. Fuzzy inference system comprises of four components:
- Fuzzifier: The fuzzifier performs the fuzzification function that converts an inputs into suitable linguistic values which are needed for the calculations by the inference engine. Input variables are minimum required bandwidth, remaining connection time, currently used bandwidth. The input variables are fuzzified by determining the degree to which they belong to each of the appropriate fuzzy set via membership functions.

- Fuzzy rule base: The fuzzy rule base is composed of a set of linguistic control rules and the attendant control goals. According to Guillaume [14], fuzzy inference system contains fuzzy rules built from expert knowledge. Based on input variables minimum required bandwidth, remaining connection time, currently used bandwidth with three linguistics, twenty nine relevant rules are created. The rules are designed by expert to describe the importance of the input variables over the possible status [17].

- Inference Engine: The inference engine simulates human decision-making, based on the fuzzy control rules and the related input linguistic parameters. 
- Defuzzifier: The defuzzifier acquires the aggregated linguistic values from the inferred fuzzy control action, and generates a non-fuzzy control output, which represents the predicted priority [18]. Fig 2 shows the fuzzy inference system.

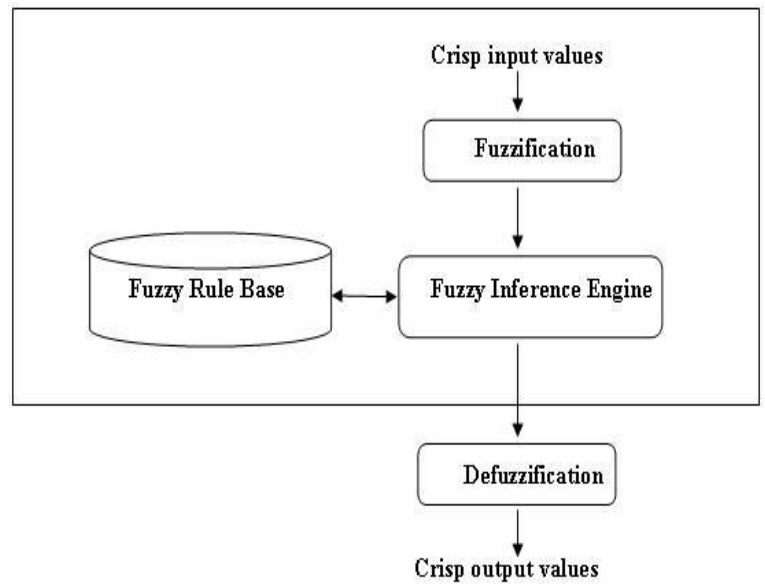

Fig2: Fuzzy Inference System [19]

\subsection{Genetic Algorithm}

Genetic Algorithms are population based search techniques that maintains the populations of potential solutions during searches. A string with a fixed bit length usually shows a potential solution. In order to calculate each potential solution, Genetic Algorithms need a payoff (or reward, objective) function that assigns scalar payoff to any particular solution. Once the representation scheme and evaluation function is determined, a Genetic Algorithm starts searching.

- Initially, often at random, Genetic Algorithm creates a certain number called the population size of strings to form the first generation.

- Next, the payoff function is used to evaluate each solution in this first generation. Better solutions obtain higher payoffs [20].

- Then, on the basis of these evaluations, some genetic operations are employed to generate the next generation.

- The procedures of evaluation and generation are iteratively performed until the optimal solution(s) is (are) found or the time allotted for computation ends [21-23]. Fig 3 shows the components of the genetic algorithm.

Before a GA can be run, it must have the following five components:

1. A chromosomal representation of solutions to the problem.

Usually, only two components of GA are problem dependent: the representation and evaluation functions. Representation is a key genetic algorithm issue because genetic algorithms directly manipulate coded representations of problems. In principle, any character set and coding scheme can be used. However, binary character set is preferred because it yield the largest number of schemata for any given parameter resolution, thereby enhancing the implicit parallelism of genetic searches.

2. A function that evaluates the performances of solutions
Along with the representation scheme, the evaluation function is problem dependent. GAs are search techniques based on feedback received from their exploration of solutions. The judge of the GA's exploration is called an evaluation function. The notion of evaluation and fitness are sometimes used interchangeably. However, it is important to distinguish between the evaluation function and the fitness function. While evaluation functions provide a measure of an individual's performance, fitness functions provide a measure of an individual's reproduction opportunities. In fact, evaluation of an individual is independent of other individuals, while an individual's fitness is always dependent of other individuals.

3. A population of initialized solutions.

Choosing an appropriate population size for a genetic algorithm is a necessary but difficult task for all GA users. On the one hand, if the population size is too small, the genetic algorithm will converge too quickly to find the optimal solution. On the other hand, if the population size is too large, the computation cost may be prohibitive.

4. Genetic operators that evolve the population.

GA is an iterative process in which each iteration has two steps, evaluation and generation. In the evaluation step, domain information is used to evaluate the quality of an individual. The generation step includes a selection phase and a recombination phase. In the selection phase, fitness is used to guide the reproduction of new candidates for following iterations. The fitness function maps an individual to a real number that is used to indicate how many offspring that individual is expected to breed. High-fitness individuals are given more emphasis in subsequent generations because they are selected more often. In the recombination phase, crossover and mutation perform mixing. Crossover reconstructs a pair of selected individuals to create two new offspring. Mutation is responsible for re-introduction inadvertently "lost" gene values. Mutation: When individuals are represented as bit strings, mutation consists of reversing a randomly chosen bit.

5. Parameters that specify the probabilities by which these genetic operators are applied.

Running a genetic algorithm entails setting a number of parameter values. However, finding good settings that work well on one's problem is not a trivial task. There are two primary parameters concern the behavior of genetic algorithms: Crossover Rate $(\mathrm{Cr})$ and Mutation Rate $(\mathrm{Mr})$.

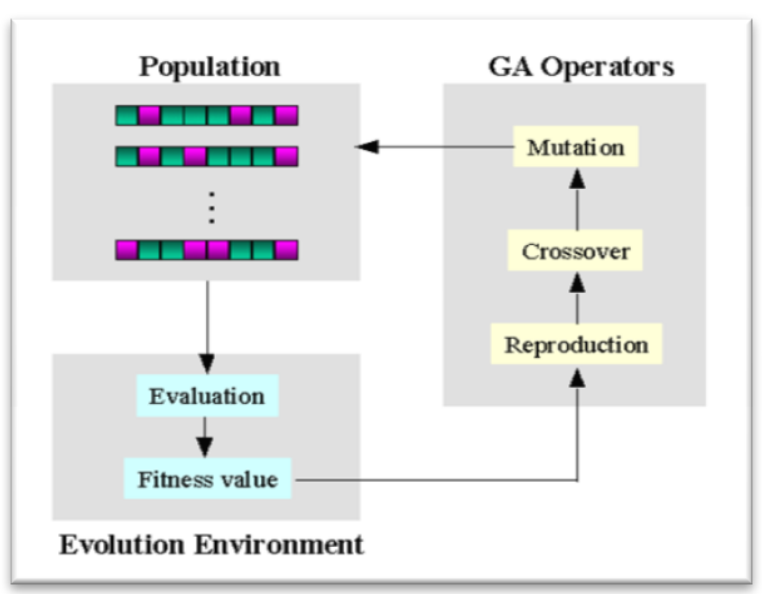


Fig3 Evolution flow of genetic algorithm [19]

\subsection{Hybrid of Fuzzy and genetic algorithm (HFGA or} FGA)

HFGA is the combination of fuzzy inference and genetic algorithm. HFGA is s scheme which is used to gain an optimal solution for data dissemination. It is used to transmit the data among the vehicle in V2V. It will choose the best vehicle to transmit the data. In thus scheme, firstly fuzzy inference system will run, after that genetic algorithm will do its work. In this scheme, genetic algorithm will use fuzzy's output as the input. After that, selection, crossover, mutation will done.

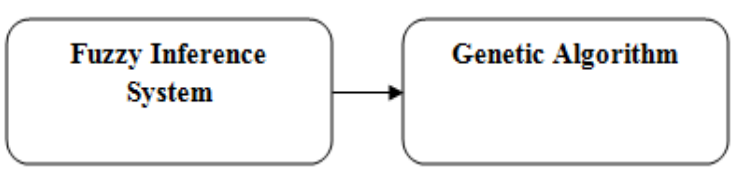

Fig4: HFGA

HFGA=Fuzzy Inference System's output----Genetic Algorithm's Input

\section{DESIGN AND IMPLEMENTATION OF HFGA}

This section explains the implementation of stepwise procedures of implementation of Fuzzy logic inference system, Genetic algorithm based system and hybrid of fuzzy logic and genetic algorithm system (HFGA or FGA). The step-wise procedures could be used to compare the among schemes. Fig5 shows the flowchart of flow of operations among the different schemes. In this schemes, there are two types of users, one is premium users and second is free users.

- Premium Users: Premium users are those users which are always accessing internet. There is bandwidth reservation mechanism for premium users to ensure the QoS of stream data which premium users received.

- Free Users: Free users are those users which help premium users to keep accessing Internet resources without interruption and degradation of the service. There is free streaming service for free users but with limited bandwidth. If there is the shortage of bandwidth, then the quality of the streaming data will be less and compression ratio, frames per second for the free users will be degraded in order to provide more bandwidth to meet the minimum bandwidth requirements for the premium users who have higher priority[5]

There are two types of zones; one is blind zone and broadcast zone.

- Blind Zone: Blind zone is that area which is not in range of base station.

- Broadcast Zone: Broadcast Zone is that area which is under the range of base station

\subsection{Fuzzy Logic Inference system}

Step 1: Generate network scenario using NS-2
Step2: Start with some initial elements like 'transmission range', 'max contention window', 'min contention window', 'neighbor list', 'forwarder vehicle', 'distance to current vehicle'

Step3: Initialize with $\mathrm{n}$ no. Of nodes where are initial values is equal to null.

Step4: Implement Protocol System.

Step5: Start Data Transmission with fuzzy inference system.

Step6: In fuzzy inference system, generate input membership function, output membership function, $\&$ rule base according to inputs and Return rules for transmission parameters.

Step7: It generates $\alpha$ feasible solutions randomly and then selects the best solutions.

Step8: Selection of best vehicle or base station for transmission and transmission done.

Step9: At last we will analysis the performance of fuzzy inference system by using the different parameters such as throughput, packet delivery ratio, and end to end delay.

\subsection{Genetic Algorithm Based System}

Step 1: Generate network scenario using NS-2

Step2: Start with some initial elements like 'transmission range', 'max contention window', 'min contention window', 'neighbor list', 'forwarder vehicle', 'distance to current vehicle'

Step3: Initialize with n no. Of nodes where are initial values is equal to null.

Step4: Implement Protocol System.

Step5: Start Data Transmission with genetic algorithm.

Step6: In genetic algorithm, firstly GA initializes the population and represent in binary form. Then fitness evaluation takes place.

Step7: After crossover \& mutation the final best solution has been selected for transmission.

Step8: Selection of best vehicle or base station for transmission and transmission done.

Step9: At last we will analysis the performance of genetic algorithm by using the different parameters such as throughput, packet delivery ratio, and end to end delay.

\subsection{HFGA (Hybrid of fuzzy inference and genetic algorithm)}

Step 1: Generate network scenario using NS-2

Step 2: Start with some initial elements like 'transmission range', 'max contention window', 'min contention window', 'neighbor list', 'forwarder vehicle', 'distance to current vehicle'

Step 3: Initialize with $\mathrm{n}$ no. Of nodes where are initial values is equal to null.

Step4: Implement Protocol System.

Step 5: Start Data Transmission with HFGA where first fuzzy inference system starts. 
Step 6: In fuzzy inference system, generate input membership function, output membership function, $\&$ rule base according to inputs and Return rules for transmission parameters.

Step 7: Then finally GA works which take output of fuzzy inference system as their input. Firstly GA Generate $\alpha$ feasible solutions randomly and then Select the best solutions. After crossover \& mutation the final best solution has been selected for transmission.
Step8: Selection of best vehicle or base station for transmission and transmission done.

Step9: At last we will analysis the performance of genetic algorithm by using the different parameters such as throughput, packet delivery ratio, and end to end delay.

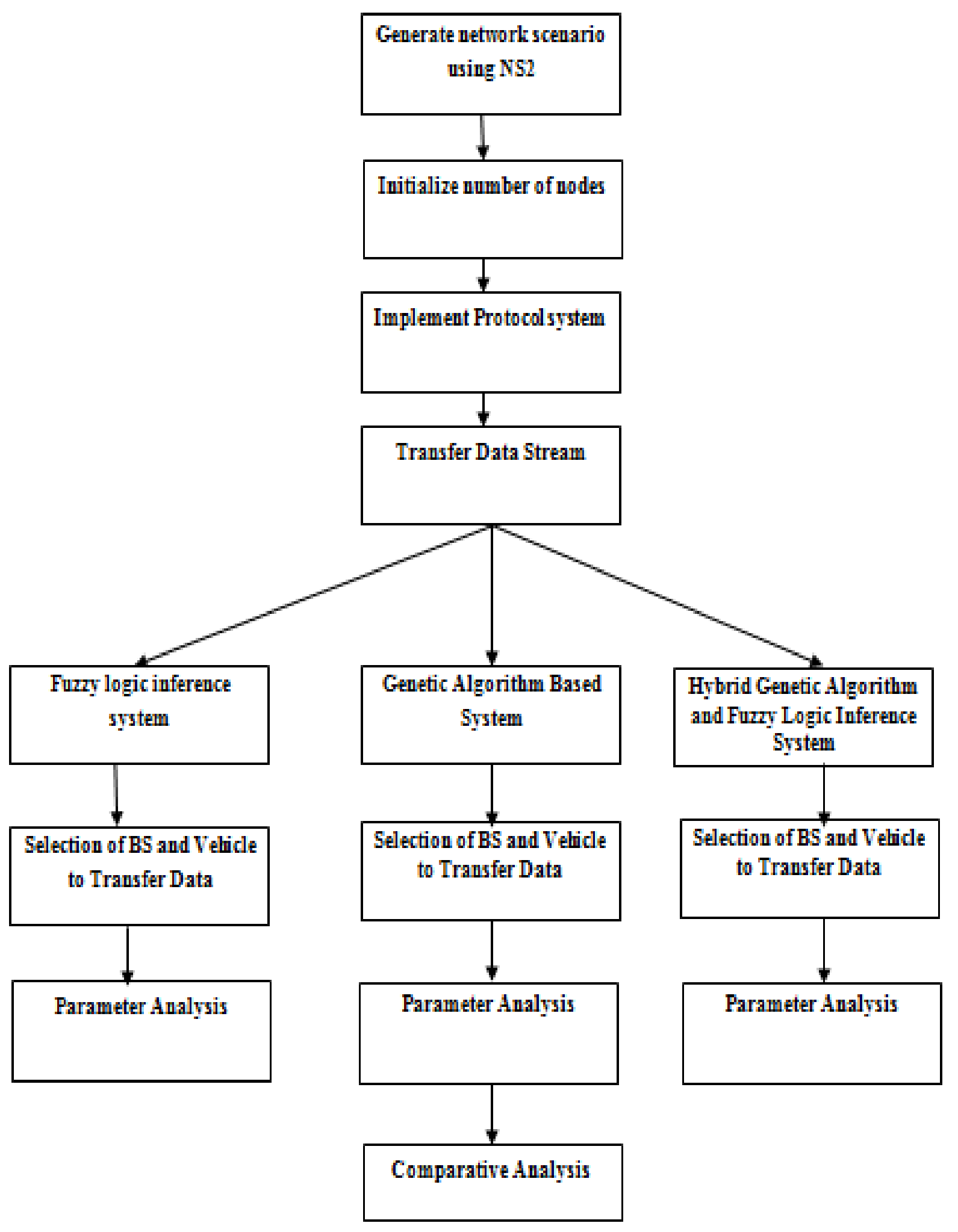


Fig5: Flow of Operations

\section{RESULT ANALYSIS}

We ran a series of simulation experiments to investigate the feasibility and effectiveness of fuzzy inference system, genetic algorithm and hybrid of fuzzy inference and genetic algorithm scheme. The simulation metrics are data delivery ratio, end to end delay and throughput among the premium users as well as free users.

- Packet delivery Ratio (PDR): It is the ratio of all the received data packets at the destination to the number of data packets sent by all the sources. It is calculated by dividing the number of packet received by destination through the no. of packet originated from the source.

$$
\mathrm{PDR}=\left(\mathrm{P}_{\mathrm{r}} / \mathrm{P}_{\mathrm{s}}\right) * 100
$$

Where, $P_{r}$ is total packet received and $P_{s}$ is total packet sent.

- End to End Delay: This includes all possible delays caused by buffering during route discovery, latency, and retransmission by intermediate nodes, processing delay and propagation delay. It is calculated as

$$
\mathrm{D}=\left(\mathrm{T}_{\mathrm{r}}-\mathrm{T}_{\mathrm{s}}\right)
$$

Where, $T_{r}$ is receive time and $T_{s}$ is sent time of the packet.

- Throughput: It is the average at which data packet is delivered successfully from one node to another over a communication network. It is usually measured in bits per second.

Throughput $=($ no of delivered packets $*$ packet size) / total duration of simulation

Experimental Setup. The experiments are performed by using the wireless channel on $2000 * 2000 \mathrm{~m}$ area. In the experiments, the initial position of all vehicles are randomly placed, and vehicles make turns at the intersections with a certain probability(turning left and turning right and going straight). The vehicle density is one of the most important factors affecting the connectivity of a VANET. The experimental results show that HFGA scheme is more reliable than others.

Result analysis of present work under different performance metrics are given in figures

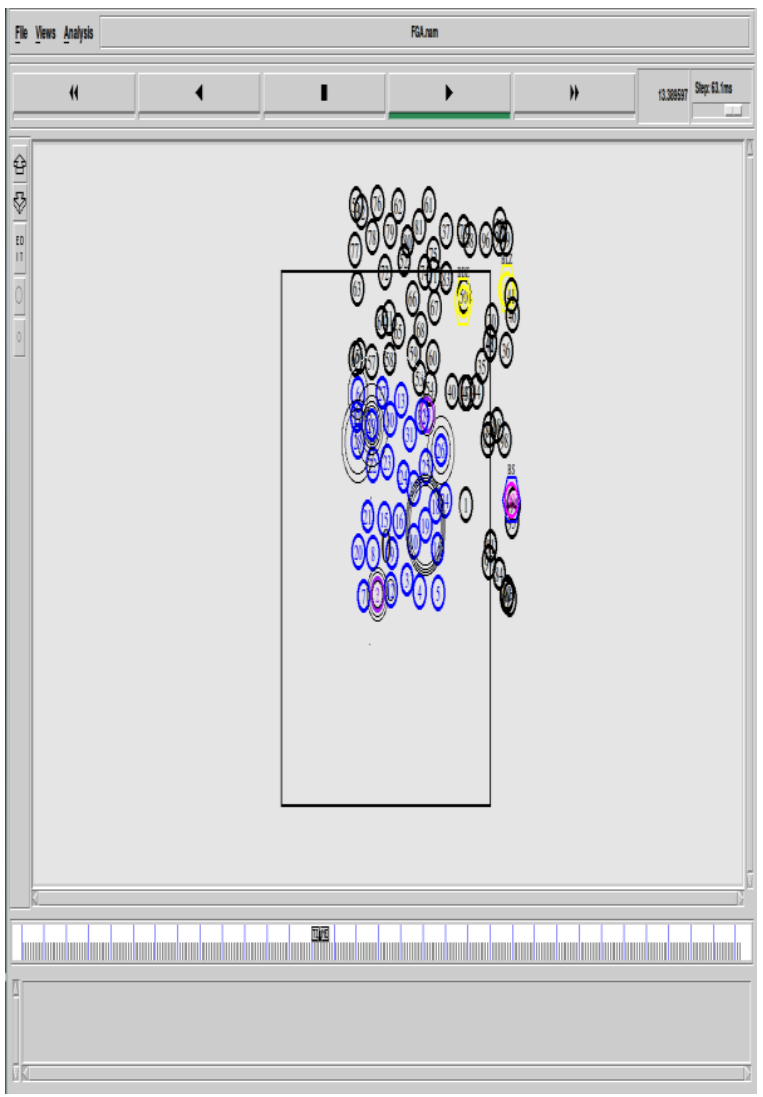

Fig 6:Generating network scenario using NS-2

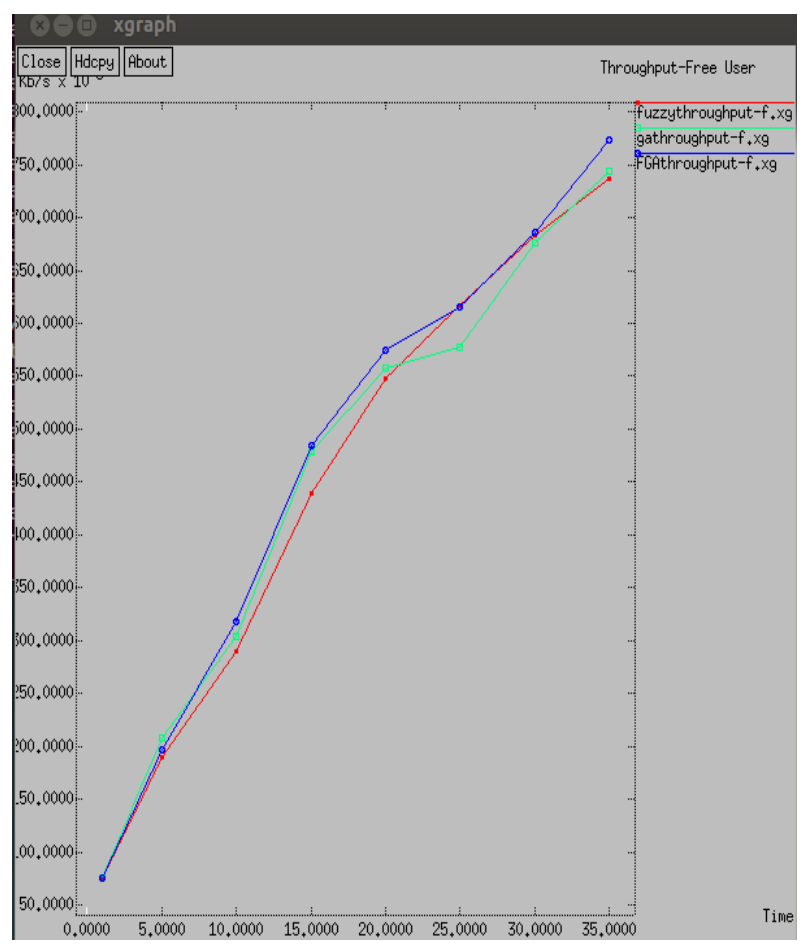

Fig7: Comparison of throughput of free users among the three schemes 
Table 1. Performance Analysis of Throughput For Free Users

\begin{tabular}{|c|c|c|c|}
\hline $\begin{array}{c}\text { Time } \\
\text { (Seconds) }\end{array}$ & $\begin{array}{c}\text { Fuzzy } \\
\text { Inference } \\
\text { System }\end{array}$ & $\begin{array}{l}\text { Genetic } \\
\text { Algorithm }\end{array}$ & $\begin{array}{c}\text { Hybrid of } \\
\text { Fuzzy and } \\
\text { Genetic } \\
\text { Algorithm }\end{array}$ \\
\hline $\mathbf{5}$ & 19.00 & 19.74 & 20.75 \\
\hline $\mathbf{2 0}$ & 54.75 & 55.75 & 57.49 \\
\hline $\mathbf{3 5}$ & 73.67 & 74.43 & 77.37 \\
\hline
\end{tabular}

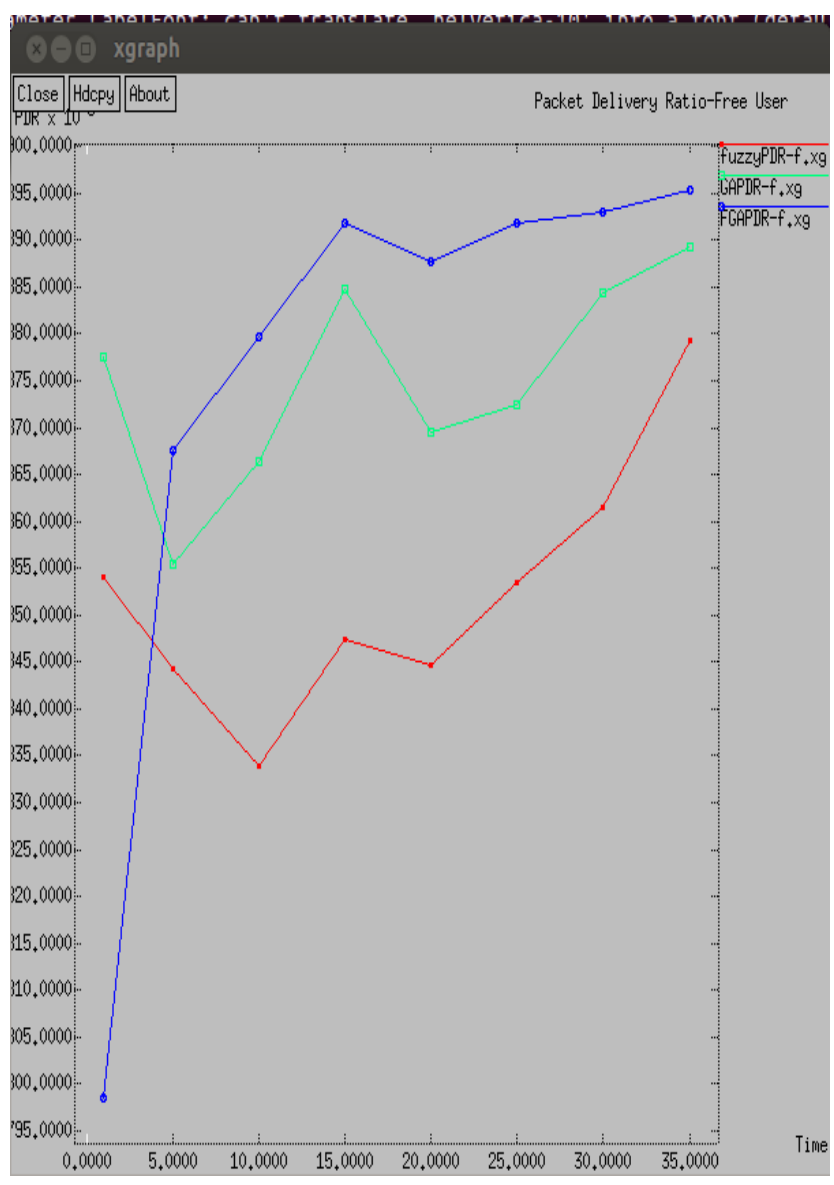

Fig8: Comparison of Packet Delivery Ratio of free users among the three schemes

Table 2. Performance Analysis Of Packet Delivery Ratio For Free Users

\begin{tabular}{|c|c|c|c|}
\hline $\begin{array}{c}\text { Time } \\
\text { (Seconds) }\end{array}$ & $\begin{array}{c}\text { Fuzzy } \\
\text { Inference } \\
\text { System }\end{array}$ & $\begin{array}{c}\text { Genetic } \\
\text { Algorithm }\end{array}$ & $\begin{array}{c}\text { Hybrid of } \\
\text { Fuzzy and } \\
\text { Genetic } \\
\text { Algorithm }\end{array}$ \\
\hline $\mathbf{5}$ & 84.44 & 8556 & 86.75 \\
\hline $\mathbf{2 0}$ & 84.46 & 8695 & 88.77 \\
\hline $\mathbf{3 5}$ & 8794 & 8893 & 8954 \\
\hline
\end{tabular}

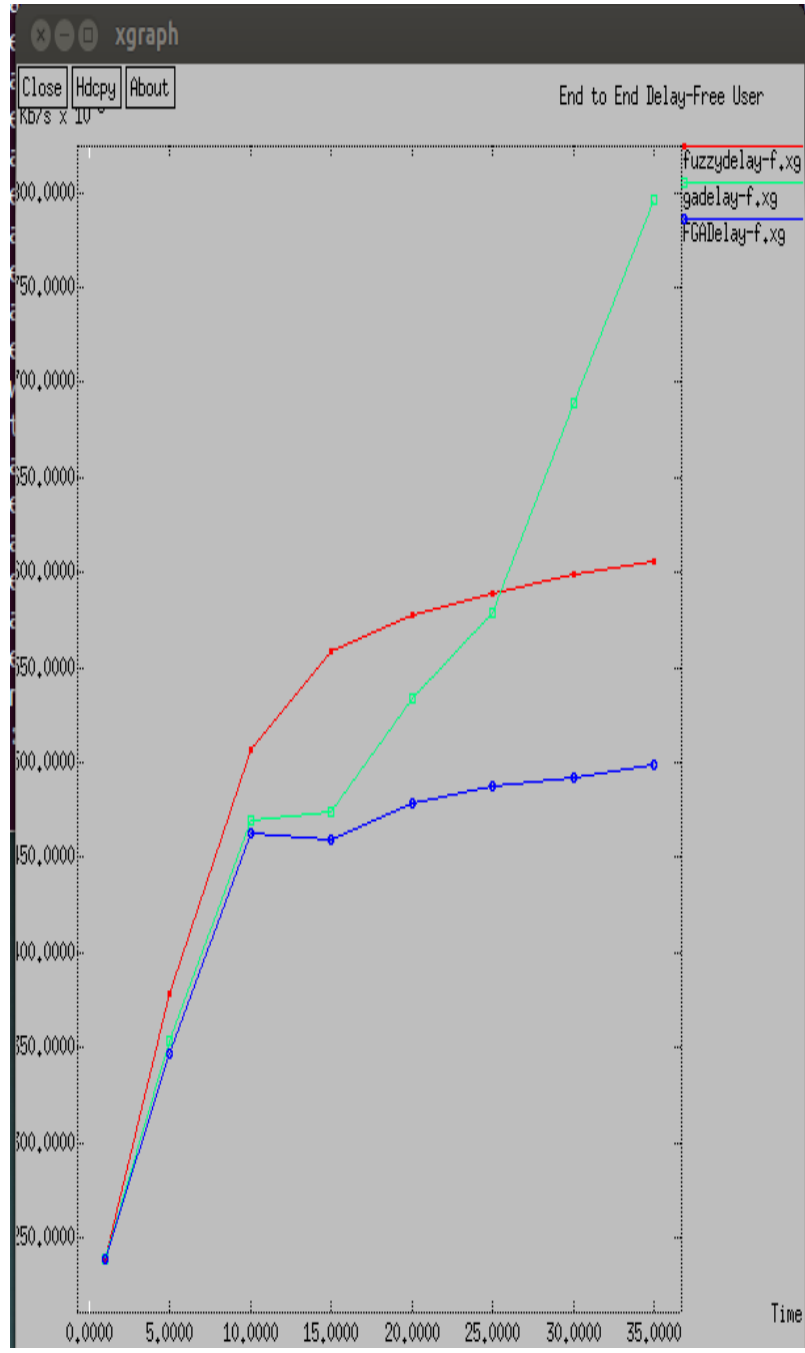

Fig9: Comparison of End to End Delay of free users among the three schemes

Table 3. Performance Analysis Of End To End Delay For Free Users

\begin{tabular}{|c|c|c|c|}
\hline $\begin{array}{c}\text { Time } \\
\text { (Seconds) }\end{array}$ & $\begin{array}{c}\text { Fuzzy } \\
\text { Inference } \\
\text { System }\end{array}$ & $\begin{array}{c}\text { Genetic } \\
\text { Algorithm }\end{array}$ & $\begin{array}{c}\text { Hybrid of } \\
\text { Fuzzy and } \\
\text { Genetic } \\
\text { Algorithm }\end{array}$ \\
\hline $\mathbf{5}$ & 37.86 & 3537 & 34.74 \\
\hline $\mathbf{2 0}$ & 57.75 & 5337 & 47.84 \\
\hline $\mathbf{3 5}$ & 60.64 & 79.64 & 49.93 \\
\hline
\end{tabular}




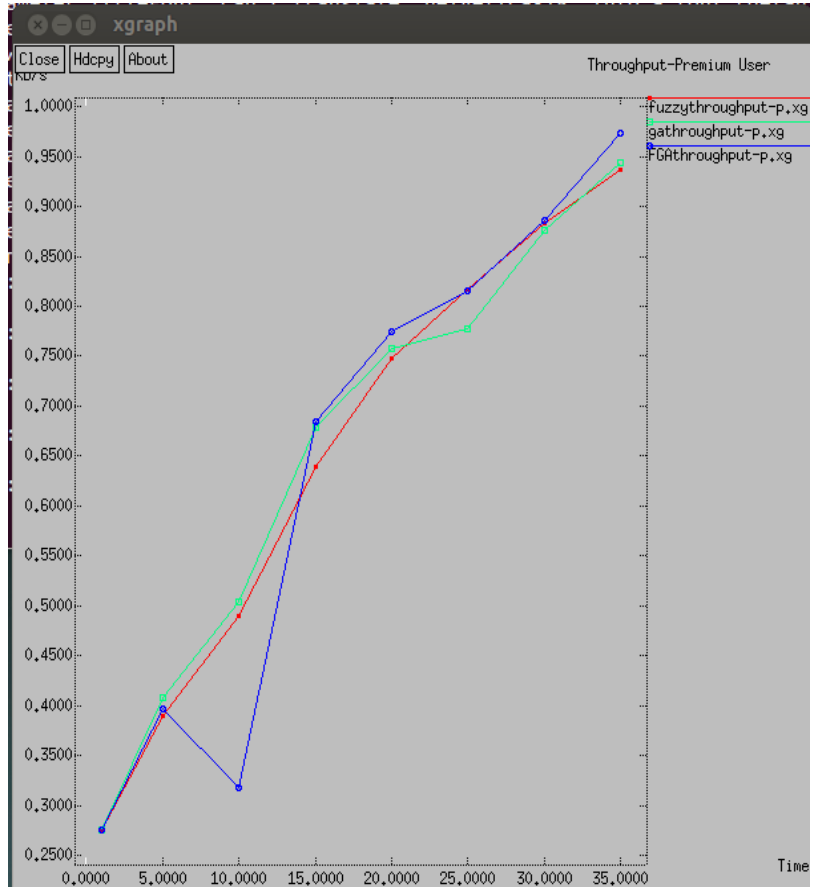

Fig10: Comparison of throughput of premium users among the three schemes

Table 4. Performance Analysis Of Throughput For Premium Users

\begin{tabular}{|c|c|c|c|}
\hline $\begin{array}{c}\text { Time } \\
\text { (Seconds) }\end{array}$ & $\begin{array}{c}\text { Fuzzy } \\
\text { Inference } \\
\text { System }\end{array}$ & $\begin{array}{c}\text { Genetic } \\
\text { Algorithm }\end{array}$ & $\begin{array}{c}\text { Hybrid of } \\
\text { Fuzzy and } \\
\text { Genetic } \\
\text { Algorithm }\end{array}$ \\
\hline $\mathbf{5}$ & 39.00 & 39.60 & 39.74 \\
\hline $\mathbf{2 0}$ & 74.75 & 75.75 & 77.49 \\
\hline $\mathbf{3 5}$ & 93.68 & 94.43 & 9737 \\
\hline
\end{tabular}

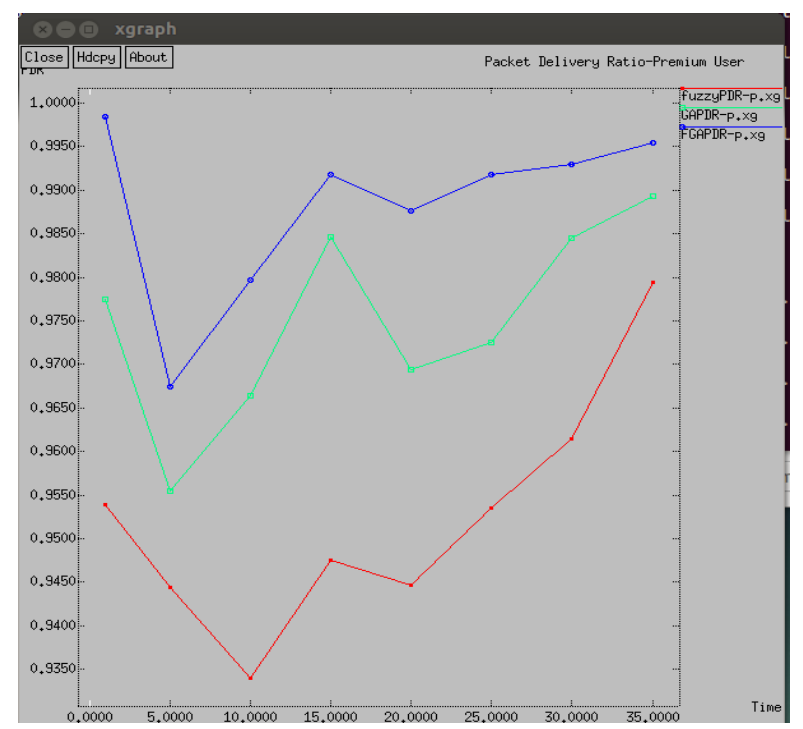

Fig11: Comparison of Packet Delivery Ratio of premium users among the three schemes
Table 5. Performance Analysis Of Packet Delivery Ratio For Premium Users

\begin{tabular}{|c|c|c|c|}
\hline $\begin{array}{c}\text { Time } \\
\text { (Seconds) }\end{array}$ & $\begin{array}{c}\text { Fuzzy } \\
\text { Inference } \\
\text { System }\end{array}$ & $\begin{array}{c}\text { Genetic } \\
\text { Algorithm }\end{array}$ & $\begin{array}{c}\text { Hybrid of } \\
\text { Fuzzy and } \\
\text { Genetic } \\
\text { Algorithm }\end{array}$ \\
\hline $\mathbf{5}$ & 94.40 & 9550 & 96.70 \\
\hline $\mathbf{2 0}$ & 9450 & 96.90 & 98.70 \\
\hline $\mathbf{3 5}$ & 87.94 & 88.93 & 8954 \\
\hline
\end{tabular}

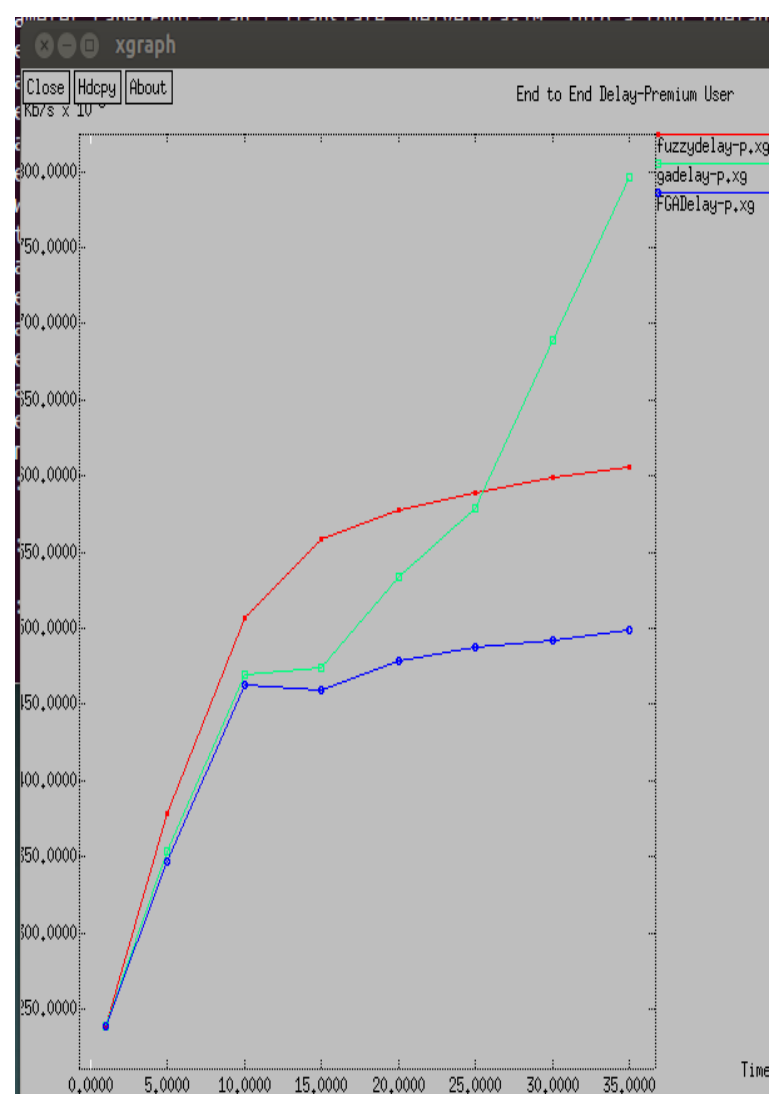

Fig12: Comparison of End to End delay of premium users among the three schemes

Table 6. Performance Analysis Of End To End Delay For Premium Users

\begin{tabular}{|c|c|c|c|}
\hline $\begin{array}{c}\text { Time } \\
\text { (Seconds) }\end{array}$ & $\begin{array}{c}\text { Fuzzy } \\
\text { Inference } \\
\text { System }\end{array}$ & $\begin{array}{c}\text { Genetic } \\
\text { Algorithm }\end{array}$ & $\begin{array}{c}\text { Hybrid of } \\
\text { Fuzzy and } \\
\text { Genetic } \\
\text { Algorithm }\end{array}$ \\
\hline $\mathbf{5}$ & 23.86 & 23.86 & 23.85 \\
\hline $\mathbf{2 0}$ & 57.75 & 5337 & 47.84 \\
\hline $\mathbf{3 5}$ & 60.64 & 79.64 & 49.93 \\
\hline
\end{tabular}

Fig 6. shows the network scenario of 100 nodes using NS-2. Fig.7 shows the throughput of free users under the condition of different numbers of vehicles among the three schemes. HFGA scheme achieves better performance in terms of throughput than the other systems and the reason is that this scheme ensures the QoS in different traffic conditions. From the results, it can observe that the throughput is slowly increasing. That is because the number of vehicles is 
increasing and limited bandwidth is being accessed by more users. However, this scheme involves using portions of free user's bandwidth to help premium users in packet transmission, and HFGA scheme also lowers free users quality in terms of compression and frame per second (fps) to this end. The result shows that the free users in HFGA scheme achieves the best performance in terms of the throughput, compared to the other schemes, and the free users in this scheme also experience better performance than the two compared methods. As shown in Fig. 8, HFGA scheme performs best in terms of packet delivery ratio. HFGA scheme will be well controlled, since this scheme considered the distribution of vehicles and BSs. When the density of vehicles and base stations is high, the system will transfer the data through selected vehicles according to the most suitable distance and velocity among the vehicles to maintain the QoS.Fig. 9 shows the end to end delay of free users among the three schemes. Notably, In HFGA scheme, although the vehicles cannot receive signals from the BS while passing out of the coverage range of the BS, they can use other vehicles to enjoy the continuously streaming for them. Therefore, the end to end delay caused by passing through areas which are outside of the coverage of any BS can be dramatically decreased. In addition, when the number of vehicles is over 100 , the end to end for free users in this scheme is slightly longer than for premier users. That is because the free users need to assist the premier users in relaying streaming data and the service disruption time for the free users is thus increased more obviously than it is for the premier users. Fig. 10 shows the throughput of premium users corresponding to the conditions of different numbers of vehicles among the three schemes. HFGA scheme proves good in terms of maximizing data throughput, with the comparisons between the three schemes. Fig. 11 shows the packet delivery ratio of the premium users. As we can see that HFGA performs best as compared to genetic algorithm and fuzzy inference system. Genetic algorithm performs better as compared to fuzzy inference system. Fig 12 shows end to end delay of premium users among the three schemes under certain conditions. As we can see from the graph that there is less delays in HFGA scheme. HFGA performs best in end to end delay case.

\section{CONCLUSION}

This paper has successfully demonstrated that the fuzzy inference scheme, genetic algorithm scheme and hybrid of fuzzy inference system and genetic algorithm scheme under certain conditions where density of base stations and vehicles both are high. This paper considers two types of users. One is premium and other is free users. The experiment was conducted to show the comparison among three selected schemes. HFGA scheme performs best in all parameters such

[2] K. N. Qureshi, A. H. Abdullah, "A survey on intelligent transportation systems," Middle-East Journal of Scientific Research, vol. 15, pp. 629-642, 2013.

[3] S. Biswas, R. Tatchikou, F. Dion, "Vehicle-to-vehicle wireless communication protocols for enhancing highway traffic safety," IEEE Communications Magazine, vol. 44, pp. 74-82, 2006. Article (CrossRef Link)

[4] IEEE P802.11 - Task Group p, "IEEE P802.11p/D9, 0, Draft Amendment for Wireless Access in Vehicular Environments (WAVE)", July 2009.

[5] Chenn-Jung Huang, Yu-Wu Wang , Heng-Ming Chen , Ai-Lin Cheng, Jui-Jiun Jian , Han-Wen Tsai , Jia-Jian as throughput, packet delivery ratio and end to end delay. It performs best in premium user's case as well as in free user's case. The HFGA provides the better way to transmit the data among the vehicles. It is demonstrated that the data dissemination among the vehicles using both fuzzy inference system and genetic algorithm is best. Playing live stream videos in vehicles thus becomes possible via VANET and roadside BSs. However, there are some difficulties to be overcome, such as the uneven distribution of roadside BSs, the high mobility of vehicles, and the volatility of network environments. In this paper, Fuzzy inference system, Genetic algorithm and HFGA for vehicular networks are explained to solve the above mentioned problems. Two users are taken under some considerations to deal with different kinds of network environments according to the number of vehicles and roadside BSs. Comparing among three schemes, the simulation results show that HFGA scheme achieves better performance in terms of throughput, packet delivery ratio and end to end delay.

\section{ACKNOWLEDGMENT}

Words are often too less to reveal ones deep regards. An understanding of the work like this is never the outcome of the efforts of single person.

I would like to express the deepest appreciation to Mr. Ayush Dhawan who has shown the attitude and the substance of a genius. He continually and persuasively conveyed a spirit of adventure in regard to research and scholarship, and an excitement in regard to teaching. Without his supervision and constant help this dissertation would not have been possible.

I am highly indebted to Dr. Tanu Preet Singh, Professor and Head of Department ECE, Amritsar College of Engineering and Technology for his guidance and constant supervision as well as for providing necessary information regarding the project $\&$ also for their support in completing this work.

I would like to express my gratitude towards my family for their kind co-operation and encouragement which help me in completion of this work.

\section{REFERENCES}

[1] Raul Amicia , Marco Bonolaa , Lorenzo Braccialea , Antonello Rabuffia , Pierpaolo Loretia , Giuseppe Bianchia , Performance assessment of an epidemic protocol in VANET using real traces ,Fourth International Conference on Selected Topics in Mobile \& Wireless Networking (MoWNet'2014) , Procedia Computer Science 40 ( 2014 ) 92 - 99.

Liao,An adaptive multimedia streaming dissemination system for vehicular networks Applied Soft Computing 13 (2013) 4508-4518

[6] Moumena Chaqfeh, Abderrahmane Lakas, Imad Jawhar ,A survey on data dissemination in vehicular ad hoc networks , Vehicular Communications 1 (2014) 214-225

[7] ] J. Zhao, G. Cao, VADD: vehicle-assisted data delivery in vehicular ad hoc networks, in: IEEE INFOCOM'06, 2006. 
[8] T. Nadeem, P. Shankar, L. Iftode, A comparative study of data dissemination models for VANETs, in: The $3^{\text {rd }}$ Annual International Conference on Mobile and Ubiquitous Systems - Workshops, 2006, pp. 1-10

[9] Annu Mor Research Scholar, Deptt. Of Computer Science Applications, Kurukshetra University, Kurukshetra,Haryana India, Study of Different Type of Data Dissemination Strategy in VANET ISSN: 23195967 ISO 9001:2008 Certified International Journal of Engineering Science and Innovative Technology (IJESIT) Volume 1, Issue 2, November 20126

[10] Pratibha Tomar, G.S tomar "state of art of data dissemination in VANETs", international journal of computer theory and engineering, vol 2, no.6 December, $20101793-8201$.

[11] IPARK Hui Zhao, Li Lu, Chao Song, and Yue Wu, : Location-Aware-Based Intelligent Parking Guidance over Infrastructureless VANETs, Hindawi Publishing Corporation International Journal of Distributed Sensor Networks Volume 2012, Article ID 280515, 12 pages doi:10.1155/2012/280515

[12] Zadeh, L.. Fuzzy sets. Information and Control $1965 ; 8: 338-353$

[13] Alicja Mieszkowicz-Rolka, Leszek Rolka, Flow graph approach for studying fuzzy inference systems, 18th International Conference on Knowledge-Based and Intelligent Information \& Engineering Systems KES2014, Procedia Computer Science 35 ( 2014 ) 681 690

[14] Guillaume, S. Designing fuzzy inference systems from data: An interpretability-oriented review. IEEE Transactions on Fuzzy Systems 2001; 9(3):426-443.

[15] Guillaume, S., Charnomordic, B. Fuzzy inference systems: An integrated modeling environment for collaboration between expert knowledge and data using FisPro. Expert Systems with Applications 2012; 39(10):8744-8755

[16] de Oliveira, J.V. Semantic constraints for membership function optimization. IEEE Transactions on Man and Cybernetics - Part A: Systems and Humans 1999; 29(1):128-138

[17] Lazim Abdullah, Modeling of health related quality of life using an integrated fuzzy inference system and linear regression, International Conference on Robot PRIDE 2013-2014 - Medical and Rehabilitation Robotics and Instrumentation, ConfPRIDE 2013-2014, Procedia Computer Science 42 (2014) 99 - 105

[18] Bhawna Dhawan, Tanu Preet Singh, Efficient Data Dissemination Techniques in VANETs: A Review, International Journal of Computer Applications (0975 8887) Volume 116 - No. 7, April 2015

[19] Philip Babatunde OSOFISAN, Department of Electrical and Electronics Engineering, University of Lagos, Akoka, Lagos, Fuzzy Logic Control of the Syrup Mixing Process in Beverage Production

[20]http://www.ewh.ieee.org/soc/es/May2001/14/Begin.htm

[21] J. H. Holland. Adaptation in Natural and Artificial Systems. The University of Michigan Press, Ann Arbor, MI, 1975

[22] J. J. Grefenstette. Optimization of control parameters for genetic algorithms. IEEE Transactions on System, Man, and Cybernetics, SMC-16(1):122-128, 1986.

[23] D. E. Goldberg. Sizing populations for serial and parallel genetic algorithms. In J. David Schaffer, editor, Proceedings of the Third International Conference on Genetic Algorithms and Their Applications, pages 7079, San Mateo, CA, June 1989. MorganKaufmann 